\title{
Intracranial Collaterals: The Next Frontier in Acute Ischemic Stroke?
}

\section{Leonard LL Yeo ${ }^{1}$ and Vijay K Sharma ${ }^{1,2^{*}}$}

${ }^{1}$ Division of Neurology, National University Hospital, Singapore

${ }^{2}$ Yong Loo Lin School of Medicine, National University of Singapore, Singapore

Intracranial arterial occlusion is responsible for the neurological deficits in Acute Ischemic Stroke (AIS). Although, timely recanalization usually results in rapid clinical recovery, this relationship is not universal and wide neurological fluctuations are often observed. Therefore, the quest for reliable biomarkers of functional outcome in AIS continues.

Intracranial collateral pathways are important for providing perfusion to the ischemic brain regions affected by an intracranial occlusion [1]. These channels may be pre-existing and compensate for the reduced perfusion via the circle of Wilis or develop de-novo. The collateral status correlates well with final infarct volume and functional outcome [2]. These collaterals can also predict the effectiveness of intravenous thrombolysis as well as various endovascular interventions [3]. Furthermore, good collaterals reduce hemorrhagic complications of various therapeutic strategies in AIS [3].

In clinical practice, infarct growth does not always progress to involve the complete vascular territory, even if the acutely occluded intracranial artery fails to recanalize. Collateral flow from various branches of the circle of Willis attempts to preserve the 'penumbra' and prevent infarct expansion. On the contrary, collateral failure is often invoked as the cause of deterioration following initial improvement, especially in patient with persistent occlusion.

Current modalities capable of imaging various intracranial collaterals in AIS include transcranial Doppler, Computerized Tomographic (CT) angiography, CT-perfusion, magnetic resonance (MR) angiography, MR-perfusion, digital subtraction angiography, positron emission tomography and single-photon emission computed tomography. These techniques have their individual advantages and limitations and often used in combination. Parenchymal perfusion imaging modalities add important information about the cerebral hemodynamics and competence of various intracranial collateral pathways. However, none of the current methods of evaluating and grading of the collaterals has demonstrated robust clinical-radiological relationship. Perhaps rapidly changing hemodynamic status in acute stroke, both natural as well as therapy induced, contributes significantly to this controversy. A rapid collateral compensatory response may be augmented by a slower response mediated by metabolic and other less well understood factors. One possible solution to solve this mystery could be the serial and multimodal vascular imaging to observe the temporal evolution and behavior of various intracranial collaterals.

Although, the clinical applications of various qualitative and quantitative collateral assessments are not established yet, rapid improvements in technology may help in future for therapeutic decision-making as well as prognostication. Newer devices for selective diversion of cerebral blood flow to enhance perfusion in AIS are already being tested in clinical trials [4]. Collateral assessment is a nascent and challenging field and rapid developments in various imaging modalities are expected to evolve as a strong tool in the armamentarium of stroke neurologists.

\section{References}

1. Liebeskind DS (2009) Stroke: the currency of collateral circulation in acute ischemic stroke. Nat Rev Neurol 5: 645-646.

2. Angermaier A, Langner S, Kirsch M, Kessler C, Hosten N, et al. (2011) CTangiographic collateralization predicts final infarct volume after intra-arteria thrombolysis for acute anterior circulation ischemic stroke. Cerebrovasc Dis 31: $177-184$

3. Calleja Al, Cortijo E, García-Bermejo P, Gómez RD, Pérez-Fernández S, et al. (2013) Collateral circulation on perfusion-computed tomography-source images predicts the response to stroke intravenous thrombolysis. Eur J Neurol 20: $795-802$

4. Liebeskind DS (2008) Aortic occlusion for cerebral ischemia: from theory to practice. Curr Cardiol Rep 10: 31-36.
*Corresponding author: Vijay K Sharma, Division of Neurology, Department of Medicine, National University Hospital, 5 Lower Kent Ridge Road, Singapore 119074, Tel: (65) 67724126; Fax: (65) 68723566; E-mail: drvijay@singnet.com.sgac.at

Received January 23, 2014; Accepted January 27, 2014; Published January 29 2014

Citation: Yeo LL, Sharma VK (2014) Intracranial Collaterals: The Next Frontier in Acute Ischemic Stroke? Brain Disord Ther 3:e109. doi:10.4172/2168-975X.1000e1089

Copyright: $\odot 2014$ Yeo LL, et al. This is an open-access article distributed under the terms of the Creative Commons Attribution License, which permits unrestricted use, distribution, and reproduction in any medium, provided the original author and source are credited. 